OPEN ACCESS

Edited by:

Eric Cox,

Ghent University, Belgium

Reviewed by:

Ashok Kumar Pandurangan,

B.S. Abdur Rahman University, India

Teresa Vezza

University of Granada, Spain

Takuya Suzuki,

Hiroshima University, Japan

${ }^{*}$ Correspondence:

Yang Tian

tianyang1208@163.com

Jun Sheng

shengjun_ynau@163.com

†These authors have contributed equally to this work

Specialty section

This article was submitted to

Mucosal Immunity,

a section of the journal

Frontiers in Immunology

Received: 01 June 2020

Accepted: 29 July 2020

Published: 15 September 2020

Citation:

Peng L, Gao X, Nie L, Xie J, Dai T,

Shi C, Tao L, Wang Y, Tian $Y$ and Sheng J (2020) Astragalin Attenuates

Dextran Sulfate Sodium

(DSS)-Induced Acute Experimental Colitis by Alleviating Gut Microbiota

Dysbiosis and Inhibiting NF- $\mathrm{kB}$ Activation in Mice.

Front. Immunol. 11:2058.

doi: 10.3389/fimmu.2020.02058

\section{Astragalin Attenuates Dextran Sulfate Sodium (DSS)-Induced Acute Experimental Colitis by Alleviating Gut Microbiota Dysbiosis and Inhibiting NF-кB Activation in Mice}

\author{
Lei Peng ${ }^{1,2,3 \dagger}$, Xiaoyu Gao 1,3,4t, Long Nie ${ }^{2 \dagger}$, Jing Xie ${ }^{1,4,5}$, Tianyi Dai ${ }^{1,4,5}$, Chongying Shi ${ }^{2}$, \\ Liang Tao ${ }^{1,2,5}$, Yan Wang ${ }^{4,5}$, Yang Tian ${ }^{1,2,4 *}$ and Jun Sheng ${ }^{3,6 *}$ \\ ${ }^{1}$ Engineering Research Center of Development and Utilization of Food and Drug Homologous Resources, Ministry of \\ Education, Yunnan Agricultural University, Kunming, China, ${ }^{2}$ College of Food Science and Technology, Yunnan Agricultural \\ University, Kunming, China, ${ }^{3}$ Yunnan Province Research Institute of Plateau Characteristic Agricultural Industry, Kunming, \\ China, ${ }^{4}$ Yunnan Provincial Key Laboratory of Biological Big Data, Yunnan Agricultural University, Kunming, China, ${ }^{5}$ Yunnan \\ Provincial Engineering Research Center for Edible and Medicinal Homologous Functional Food, Yunnan Agricultural \\ University, Kunming, China, ${ }^{6}$ Key Laboratory of Pu-er Tea Science, Ministry of Education, Yunnan Agricultural University, \\ Kunming, China
}

With the ulcerative colitis $(\mathrm{UC})$ incidence increasing worldwide, it is of great importance to prevent and treat UC. However, efficient treatment options for UC are relatively limited. Due to the potentially serious adverse effects of existing drugs, there is an increasing demand for alternative candidate resources derived from natural and functional foods. Astragalin (AG) is a type of anti-inflammatory flavonoid, with Moringa oleifera and Cassia alata being its main sources. In this study, we investigated the therapeutic effects of AG on mice with dextran sulfate sodium (DSS)-induced colitis. Our results suggested that $A G$ treatment reduced weight loss and the disease activity index (DAl), prevented colon shortening and alleviated colonic tissue damage. AG treatment reduced the expression of pro-inflammatory cytokines and related mRNAs (such as TNF- $\alpha, I L-6$, and $(L-1 \beta)$, inhibited colonic infiltration by macrophages and neutrophils, ameliorated metabolic endotoxemia, and improved intestinal mucosal barrier function (increased expression levels of mRNAs such as ZO-1, occludin, and Muc2). Western blot analysis revealed that AG downregulated the NF-кB signaling pathway. Moreover, AG treatment partially reversed the alterations in the gut microbiota in colitis mice, mainly by increasing the abundance of potentially beneficial bacteria (such as Ruminococcaceae) and decreasing the abundance of potentially harmful bacteria (such as Escherichia-Shigella). Ruminococcaceae and Enterobacteriaceae (Escherichia-Shigella) were thought to be the key groups affected by AG to improve UC. Therefore, AG might exert a good antiUC effect through microbiota/LPS/TLR4/NF-kB-related pathways in mice. The results of this study reveal the anti-inflammatory effect and mechanism of $A G$ and provide an important reference for studying the mechanisms of natural flavonoids involved in preventing inflammation-driven diseases.

Keywords: Astragalin, Escherichia-Shigella, gut microbiota, NF-кB, Ruminococcaceae, ulcerative colitis 


\section{INTRODUCTION}

Ulcerative colitis (UC) is one of the main types of inflammatory bowel disease (IBD) and has an increasing incidence worldwide. Its pathogenesis is multifactorial, including genetic susceptibility, epithelial barrier defects, changes in the intestinal microbiota, immune dysregulation, and environmental factors $(1,2)$.

In recent years, the intestinal microbiota has been widely recognized to regulate intestinal homeostasis and the pathogenesis of UC. Changes and disorder in the gut microbiota are closely related to the occurrence or development of UC, but whether disorder is primary or secondary is unclear (3). The mechanisms involved are still not fully understood.

Currently, 5-aminosalicylate (5-ASA), glucocorticosteroids, azathioprine and cyclosporine are commonly used drugs for UC treatment (4). Unfortunately, the long-term use of these drugs has been found to cause severe toxicity; therefore, it is increasingly necessary to explore alternative candidate agents derived from natural resources and functional foods for UC.

Astragalin (AG), a natural flavonoid, has been found in various traditional medicinal and edible plants, such as Moringa oleifera, Radix astragali, Morus alba, and Cassia alata. AG exhibits various pharmacological properties, including anti-inflammatory, antioxidant, neurological, cardioprotective, antidiabetic, and anticancer effects (5). Previous studies on the anti-inflammatory activity of AG in animals have mainly reported the effects of $A G$ on mastitis, endotoxemia, lung injury, allergic inflammation and hepatic fibrosis in mice or rats (5). Astragalin has been reported to modulate inflammatory responses by regulating the expression of NF- $\mathrm{kB}$, iNOS, cytokines/chemokines, MAPK signaling pathway components, and PAR2 signaling pathway components (6). However, to date, there have been no reports on the treatment effects of $A G$ on UC and the gut microbiota. Most flavonoids or polyphenols have low bioavailability and a low absorption rate, and up to $90 \%$ of these compounds remain in the colon (7). Polyphenols such as resveratrol, epigallocatechin-3-gallate, curcumin, quercetin, and anthocyanin have been verified to have therapeutic roles in IBD animal models (8-12). Their therapeutic functions in IBD usually include regulating the NF- $\mathrm{KB}$ pathway and gut barrier function, and a large number of studies have shown that the gut microbiota is also an important target (13). This finding provides a theoretical basis for the hypothesis that AG and its metabolites may alleviate UC by regulating the gut microbiota.

Here, we evaluated the anti-UC activity of AG in a dextran sodium sulfate (DSS)-induced UC mouse model. Ultimately, the anti-UC effects and mechanisms of AG related to antiinflammatory activity, NF- $\mathrm{kB}$ signaling pathway downregulation, intestinal barrier improvement, and gut microbiota regulation were evaluated.

\section{MATERIALS AND METHODS}

\section{Reagents}

AG (purity > 98\%) was purchased from Chengdu Derick Biotechnology Co., Ltd. (Chengdu, China). AG is difficult to dissolve in water (or PBS), so we dissolved it in DMSO. DSS
(36,000 to $50,000 \mathrm{Da}$ ) was purchased from MP Biomedicals (Solon, OH, USA). 5-ASA and RIPA cell lysis buffer containing $1 \%$ phenylmethanesulfonyl fluoride (PMSF) were obtained from Solarbio (Beijing, China). A myeloperoxidase (MPO) detection kit was obtained from Nanjing Jiancheng Bioengineering Institute. Antibodies against $\beta$-actin (\#4970), IкB $\alpha(\# 4812)$, phospho-IкB $\alpha$ (\#4792), p65 (\#5970), phospho-p65 (\#3031), IKK $\alpha$ (\#61294), IKK $\beta$ (\#8943), and p-IKK $\alpha / \beta$ (\#2697) were purchased from CST (Boston, MA, USA). An anti-rabbit IgG antibody was purchased from R\&D Systems (Minneapolis, MN, USA).

\section{Animal Experimental Design}

The experimental protocols were approved by the Yunnan Agricultural University Animal Ethics Committee with respect to ethical issues and scientific care (YNAU-2018-031).

Male C57BL/6J mice (6 weeks old, 17-21 grams, $n=56$ ) were purchased from the Model Animal Research Center of Nanjing University (Nanjing, China) and housed under constant conditions (room temperature $24 \pm 1^{\circ} \mathrm{C}, 12 \mathrm{~h} \mathrm{light/dark} \mathrm{cycle,}$ lights off at 20:00) with clean water and the AIN-93M diet (Trophic Animal Feed High-tech Co., Ltd., Nantong, China) available ad libitum. After 1 week of acclimation, the mice were randomly divided into seven groups of 8 mice each according to their body weights as follows: a control group (received water as vehicle); DSS + PBS group; DSS + DMSO group; DSS + 50 $\mathrm{mg} / \mathrm{kg}$ AG group (DSS + AG50); DSS + $75 \mathrm{mg} / \mathrm{kg}$ AG group (DSS + AG75); DSS + $100 \mathrm{mg} / \mathrm{kg}$ AG group (DSS + AG100); and DSS $+50 \mathrm{mg} / \mathrm{kg}$ 5-ASA group (DSS + 5-ASA). AG was dissolved in DMSO. The DSS + DMSO group was the solvent control. The DSS + PBS group was set up to exclude any unknown effects of the solvent DMSO on the experimental results. A DSS-induced UC mouse model was generated as previously described (14). Briefly, the colitis was induced by administration of 3\% DSS for 7 days, and at the same time, the mice were gavaged with daily oral doses of $200 \mu \mathrm{L}$ of different solutions. Different doses of AG were dissolved in $20 \mu \mathrm{L}$ of DMSO $+180 \mu \mathrm{L}$ of PBS. All the mice were freely allowed normal water for 2 more days to observe the development of colitis (Figure 1A).

Body weight changes and the disease activity index (DAI) were recorded during the entire animal experiment. The DAI is the sum of scores for weight loss, stool consistency, and the gross bleeding extent. The detailed scoring criteria for the DAI (15) are described in Table S1. After $12 \mathrm{~h}$ of fasting, the mice were sacrificed by $\mathrm{CO}_{2}$ asphyxiation at the end of the experimental period.

\section{Histological Assessment}

Colon tissue samples were fixed in $10 \%$ formalin and then embedded in paraffin. All tissue samples were cut into $3 \mu \mathrm{m}$ sections for staining with hematoxylin and eosin (H\&E). All H\&E-stained tissue sections were assessed using the following scoring scheme (16), including inflammatory infiltration (0$5)$, crypt injury (0-4), ulcer (0-3), and presence or absence of edema (0 or 1). All histological scoring was performed by 2 blinded investigators. 
A

B

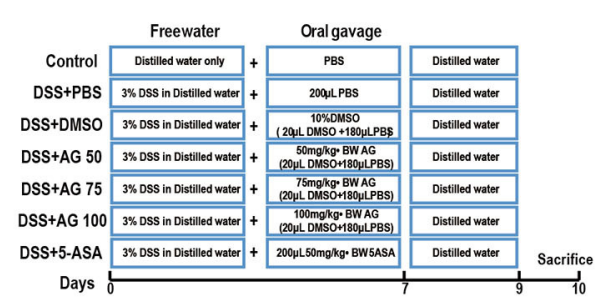

D

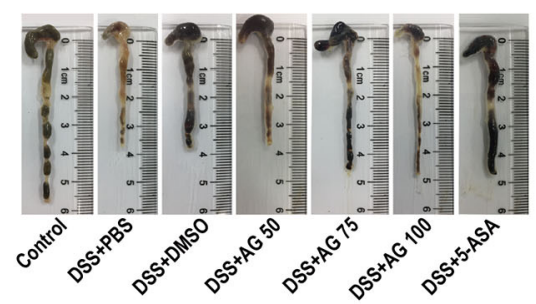

E

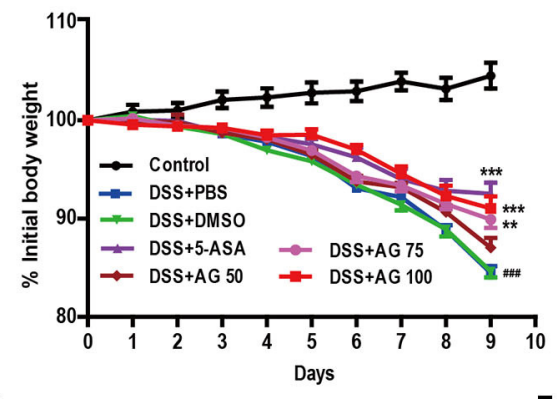

$F$

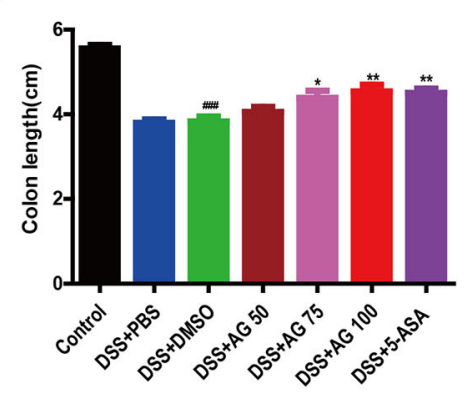

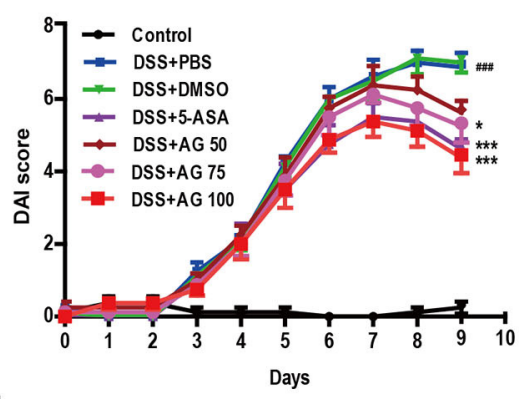

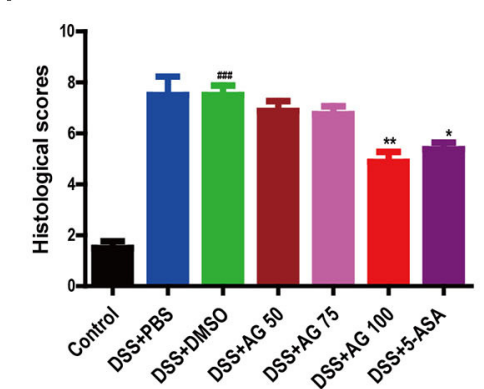

G
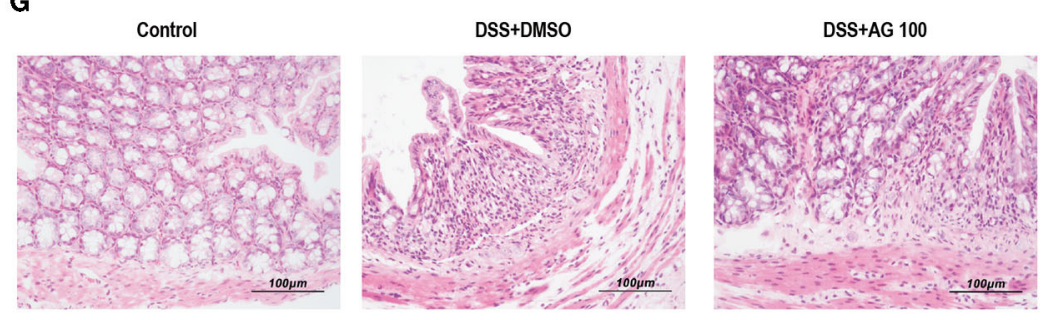

FIGURE 1 | AG attenuates symptoms of DSS-induced colitis in mice. (A) Schematic diagram of the animal experimental design. (B) Mouse body weights measured daily. (C) Calculated DAl scores. (D,E) Intestine images and statistics for colon length in each group. (F) Histological scores of colonic tissue. (G) Representative HandE-stained images of colon sections (400x magnification). Data are expressed as the mean \pm SEM, $n=8$. Data were analyzed using one-way ANOVA (post hoc Tukey's multiple-comparison test). $\# \# \# P<0.001$ compared with the control group. ${ }^{*} P<0.05$, ${ }^{* \star} P<0.01$, and ${ }^{\star \star \star} P<0.001$ compared with the DSS + DMSO group.

\section{Immunohistochemistry and an Immunoblotting Assay}

Immunohistochemistry was performed using a previously described method (17). Briefly, $3 \mu \mathrm{m}$ sections were deparaffinized in xylene and rehydrated in graded alcohol. After quenching endogenous peroxidase activity and blocking non-specific binding, the sections were incubated with a mouse monoclonal antibody against CD177 or F4/80 (Santa Cruz, CA, USA) overnight at $4^{\circ} \mathrm{C}$ and then incubated with a biotinylated secondary antibody (Zymed Laboratories, Carlsbad, CA, USA) at room temperature for $20 \mathrm{~min}$. Finally, the slides were incubated with reagents from the Avidin-Biotin Complex Kit (Vector Laboratories, Burlingame, CA, USA) and a 3,3'diaminobenzidine kit (Tiangen, Sichuan, China) according to the manufacturer's instructions. Images were captured with an Olympus CX43 microscope and CellSens Entry software.

\section{Real-Time PCR Analysis}

Total RNA was extracted from colonic samples using TRIzol (TransGen Biotech, Beijing, China) according to the manufacturer's protocol and quantified using a NanoDrop 2000 Spectrophotometer (Thermo Fisher Scientific Inc., Waltham, MA, USA). Complementary DNA was synthesized using a PrimeScript RT reagent kit with genomic DNA eraser (Takara, Dalian, China) in accordance with the manufacturer's protocol. Quantitative real-time polymerase chain reaction (RT-qPCR) was performed with SYBR ${ }^{\circledR}$ Premix Ex Taq $^{\mathrm{TM}}$ II (TliRNaseH Plus, Takara, Dalian, China) and the ABI 7900HT Fast Real-Time PCR System (Applied Biosystems, Inc.). The relative amount of the target mRNA was normalized to the $\beta$-actin level, and results were calculated by the $2^{-\Delta \Delta C t}$ method. The specific primers used are indicated in Table S2. 


\section{Enzyme-Linked Immunosorbent Assay (ELISA)}

Colon tissue samples were homogenized in a buffer containing protease inhibitors for $1 \mathrm{~min}$. The mixture was incubated on ice for $15 \mathrm{~min}$ and then centrifuged at $15,000 \mathrm{~g}$ for $10 \mathrm{~min}$ to obtain the supernatant. The concentrations of monocyte chemoattractant protein 1 (MCP-1), tumor necrosis factor alpha (TNF- $\alpha$ ), IL-1 beta (IL-1 $\beta$ ), and interleukin 6 (IL-6) in the supernatants of the colon tissue samples were measured using ELISA kits (Beijing 4A Biotech Co., Ltd., Beijing, China) according to the protocol of the manufacturer. A Cusabio ELISA kit (Wuhan, China) was used to determine the serum LPS concentration.

\section{MPO Activity}

Colon tissue samples from approximately the same site were weighed and homogenized in ice-cold PBS. MPO activity was measured using a kit according to the manufacturer's instructions. MPO activity was measured using the $\mathrm{O}$-dianisidine method and is reported as units per gram of wet tissue.

\section{Western Blot Analysis}

Colon tissue samples were minced and placed in Eppendorf tubes. RIPA buffer (Solarbio Life Science, Beijing, China) supplemented with a protease and $1 \%$ PMSF was used to isolate total protein from the colon tissue samples. A BCA protein kit (Beyotime Biotechnology, China) was used to determine the protein concentration. Whole amounts of protein $(60 \mu \mathrm{g})$ were loaded onto a $10 \%$ sodium dodecyl sulfate polyacrylamide gel. Membranes were blocked with $5 \%$ bovine serum albumin after the proteins were transferred to PVDF membranes (Millipore, MA, USA). Then, the membranes were incubated overnight at $4{ }^{\circ} \mathrm{C}$ with the following primary antibodies: anti- $\beta$-actin, anti-I $\mathrm{B} \alpha$, anti-p-I $\mathrm{B} \alpha$, anti-IKK $\alpha$, anti-IKK $\beta$, anti-p-IKK $\alpha / \beta$, anti-p65, and anti-p-p65. The next day, the membranes were washed with PBST buffer and incubated with appropriately diluted horseradish peroxidase-conjugated secondary antibodies for $1 \mathrm{~h}$ at room temperature. After the membranes were washed again 3 times, the protein bands were visualized using an enhanced chemiluminescence kit (Tiangen Biotech, Beijing, China) according to the manufacturer's instructions.

\section{Sequencing of the 16S rRNA Genes of the Gut Microbiota and Bioinformatic Analysis}

Collected mouse cecal contents were stored at $-80^{\circ} \mathrm{C}$ after being snap frozen in liquid nitrogen. The cecal content samples were sent to Majorbio Biotechnology Co., Ltd. (Shanghai, China) under dry ice conditions for $16 \mathrm{~S}$ rRNA gene sequencing. Microbial DNA was extracted from the mouse cecal contents of the control group, the DSS + DMSO group and the DSS + AG 100 group using the E.Z.N.A. ${ }^{\circledR}$ soil DNA Kit (Omega Bio-tek, Norcross, GA, USA) according to the manufacturer's protocols. The final DNA concentration and purity were determined with a NanoDrop 2000 UV-Vis spectrophotometer (Thermo Scientific, Wilmington, USA), and DNA quality was checked by $1 \%$ agarose gel electrophoresis. The V3-V4 hypervariable regions of the bacterial $16 \mathrm{~S}$ rRNA gene were amplified with the primers 338F (5'- ACTCCTACGGGAGGCAGCAG$\left.3^{\prime}\right)$ and 806R (5'-GGACTACHVGGGTWTCTAAT-3') using a thermocycler PCR system (GeneAmp 9700, ABI, USA). Detailed information on the PCR procedures, purification and quantification methods of the PCR products are described in the online Supplementary Materials.

Purified amplicons were pooled in equimolar amounts and paired-end sequenced $(2 \times 300)$ on an Illumina MiSeq platform (Illumina, San Diego, CA, USA). The taxonomy of each $16 \mathrm{~S}$ rRNA gene sequence was analyzed by the RDP Classifier algorithm against the Silva (SSU123) 16S rRNA database using a confidence threshold of 70\%. Subsequent bioinformatic analysis was performed on the cloud platform of Majorbio Bio-Pharm Technology Co., Ltd. (Shanghai, China).

\section{Statistical Analysis}

Data are presented as the arithmetic mean \pm standard error of the mean (SEM). Data were analyzed using GraphPad Prism 5.0 (GraphPad, San Diego, CA, USA). Statistically significant differences between groups were evaluated by oneway analysis of variance (ANOVA) followed by Tukey's honest significant difference test. Bivariate correlations were calculated using Pearson $r$ coefficients. HemI 1.0 software was used to construct heatmaps.

\section{RESULTS}

\section{AG Inhibited DSS-Induced Colitis Symptoms}

A schematic of the animal experimental design is shown in Figure 1A. The body weight of the mice in the DSS + PBS group and the DSS + DMSO group decreased throughout the animal experiment, while the body weight of the mice in the control group increased. The inhibition of weight loss in the DSS + AG groups $(75 \mathrm{mg} / \mathrm{kg}$ and $100 \mathrm{mg} / \mathrm{kg}$ ) was comparable to that observed in the DSS + 5-ASA (50 mg/kg, positive drug control) group (Figure 1B).

The DAI score increased significantly after DSS intake, whereas it was markedly attenuated in the DSS + AG groups (75 $\mathrm{mg} / \mathrm{kg}$ and $100 \mathrm{mg} / \mathrm{kg}$ ) and the DSS + 5-ASA group (Figure 1C). Moreover, our results indicated a significant shortening of the colon in the DSS + DMSO group compared with the control group, the DSS + AG groups (75 mg/kg and $100 \mathrm{mg} / \mathrm{kg})$, and the DSS +5 -ASA group $(P<0.05$, Figures 1D,E).

Compared with that in the mice in the DSS + PBS group and the DSS + DMSO group, the colon damage in the mice in the groups treated with AG or 5-ASA was significantly reduced, including improved histological structure, reduced epithelial disintegration, and reduced inflammatory cell infiltration (Figures 1F,G, Figure S1A).

\section{Effects of AG on Colon Pro-inflammatory Factors and the Colonic Infiltration of Inflammatory Cells in DSS-Treated Mice}

Compared with those in the control group, the relative mRNA expression levels of pro-inflammatory factors, such as $M C P-1$, 
TNF- $\alpha, I L-6, I L-1 \beta$, IFN- $\gamma$, and cyclooxygenase-2 (COX-2), in the DSS-treated group were significantly enhanced (Figures 2A-F). Similar to 5-ASA, AG (100 mg/kg.BW) significantly suppressed the relative mRNA expression levels of these factors. Compared with those in the control group, the levels of all pro-inflammatory cytokines (MCP-1, TNF- $\alpha$, IL-1 $\beta$, and IL-6) in the DSS + DMSO group were increased significantly (Figures 2G-J). After treatment with $100 \mathrm{mg} / \mathrm{kg} \cdot \mathrm{BW}$ AG, the levels of proinflammatory cytokines were reduced significantly.

The results further showed that the MPO activity in the DSS + DMSO group was increased significantly (Figure 2K). Compared with that in the DSS + DMSO group, the content of MPO in the DSS + AG50 group (50 mg/kg.BW) presented no obvious change, but the DSS + AG groups (75 and $100 \mathrm{mg} / \mathrm{kg} \cdot \mathrm{BW}$ ) and the DSS + 5-ASA group generally showed an attenuation of the increasing MPO activity. Targeting MPO may mitigate oxidative damage to host tissue and ensuing inflammation.

Compared with control treatment, DSS caused increased infiltration of $\mathrm{CD}_{177^{+}}$neutrophils (Figure 2L and Figure S1B) and $\mathrm{F} 4 / 80^{+}$macrophages (Figure $2 \mathrm{M}$ and Figure S1C). The DSS + AG 100 and DSS + 5-ASA groups showed reduced infiltration of $\mathrm{CD} 177^{+}$neutrophils and $\mathrm{F} 4 / 80^{+}$macrophages. In summary, AG treatment may inhibit the infiltration of neutrophils and macrophages into the colonic lesional area, thereby reducing the severity of complications caused by UC.

\section{AG Ameliorated Metabolic Endotoxemia and Improved Intestinal Mucosal Barrier Function}

Compared with those in the control group, the mice in the DSS + PBS group and the DSS + DMSO group had significantly higher blood serum levels of lipopolysaccharide (LPS), indicating that DSS triggered metabolic endotoxemia. In the AG treatment groups, the LPS levels were lower than those in the DSS + PBS group and the DSS + DMSO group (Figure 3A) and close to those in the control group. AG (100 mg/kg.BW) significantly decreased the LPS content in the serum and ameliorated endotoxemia.

Compared with DSS + DMSO, AG significantly suppressed the relative mRNA expression levels of Toll-like receptor 4 (TLR4) and elevated the mRNA expression of ZO-1, Occludin, and $M u c 2$ (Figures 3B-E). In general, AG regulated the expression of these genes, maintaining them at levels close to those in the control group, and effectively alleviated DSS-induced metabolic endotoxemia and intestinal barrier damage.

\section{AG Inhibited NF-кB Pathway Activation}

As shown in Figure 4, compared with control treatment, DSS significantly upregulated the expression of $\mathrm{p}-\mathrm{I} \kappa \mathrm{B} \alpha, \mathrm{p}-\mathrm{IKK} \alpha / \beta$, and p-p65 in the colon of the mice in the DSS + PBS group and the DSS + DMSO group $(P<0.05)$. Similar to 5-ASA, AG $(100 \mathrm{mg} / \mathrm{kg} \cdot \mathrm{BW})$ significantly downregulated the expression of $\mathrm{p}$-I $\kappa \mathrm{B} \alpha, \mathrm{p}-\mathrm{IKK} \alpha / \beta$, and p-p65 compared with DSS + DMSO $(P<0.05$, Figures $4 \mathrm{~A}-\mathrm{D})$. The ratio of target phosphorylated protein expression to corresponding total protein expression was calculated as the protein expression level (Figures 4B-E). In summary, AG suppressed the production of pro-inflammatory cytokines by blocking the NF-кB pathway.

\section{AG Altered the Gut Microbiota in Colitis Mice}

The V3-V4 regions of the $16 \mathrm{~S}$ rRNA gene were sequenced to evaluate the effects of AG $(100 \mathrm{mg} / \mathrm{kg} \cdot \mathrm{BW})$ on DSS-induced UC mice. We obtained 1,026,250 sequences from all 18 samples from the control, DSS + DMSO and DSS + AG100 groups, each with more than 30,379 valid sequences for subsequent taxonomic analysis.

The rarefaction curve of the Sobs index of each sample plateau (Figure 5A) with the current sequencing indicates that the sequencing depth was sufficient to reflect the diversity of the sample, and the sequencing result was credible. The alpha diversity of a sample reflects the richness and diversity of the microbial community. The community richness (Sobs, Chao and Ace), community diversity (Shannon) and community evenness (Shannoneven) indices were all significantly reduced in the DSS + DMSO group compared with the control group (Figure 5B and Figures S2A-S2D, $P<0.05)$. AG (100 $\mathrm{mg} / \mathrm{kg} \cdot \mathrm{BW}$ ) administration reversed these DSS-induced diversity index changes to varying degrees. Notably, AG $(100 \mathrm{mg} / \mathrm{kg} \cdot \mathrm{BW})$ administration significantly improved the DSS-induced decrease in the Sobs index $(P<0.05$, Figure 5B). These results indicated that AG treatment could increase the gut microbiota diversity of DSS-induced UC mice. The beta diversity is displayed in Figure 5C. Principal coordinate analysis (PCoA) results showed that DSS changed the gut microbial structure significantly. Although the administration of high-dose AG (100 mg/kg.BW) could not completely reverse the effect on the gut microbiota, AG still appeared to regulate the abnormal gut microbiota in DSS-induced UC mice.

Histograms were used to indicate the relative abundances of gut microbial species in different groups. Compared with control treatment, DSS treatment increased the relative abundance of Proteobacteria and decreased the relative abundance of Verrucomicrobia, whereas AG treatment reversed these changes (Figure 5D); however, the differences were not significant (Figure 5D, Figures S2E,S2F). Figure S2G and Figure 5E show the changes in the main microbiota at the family level. The relative abundances of Prevotellaceae, Erysipelotrichaceae, Bacteroidaceae, Peptostreptococcaceae, and norank_o__Rhodospirillales were significantly increased in the DSS + DMSO group compared with the control group, whereas AG treatment reversed these changes to various degrees. In contrast, DSS decreased the relative abundances of the Ruminococcaceae and Lachnospiraceae families, while AG treatment reversed these changes. Compared with control treatment, DSS treatment significantly reduced the relative abundances of most genera belonging to the Ruminococcaceae family, whereas AG treatment significantly reversed most of these changes (Figure 5F). Notably, DSS treatment significantly increased the relative abundance of the genus Ruminococcus_1, whereas AG treatment reduced the relative abundance to a level close to that seen in the control group. Most genera belonging 

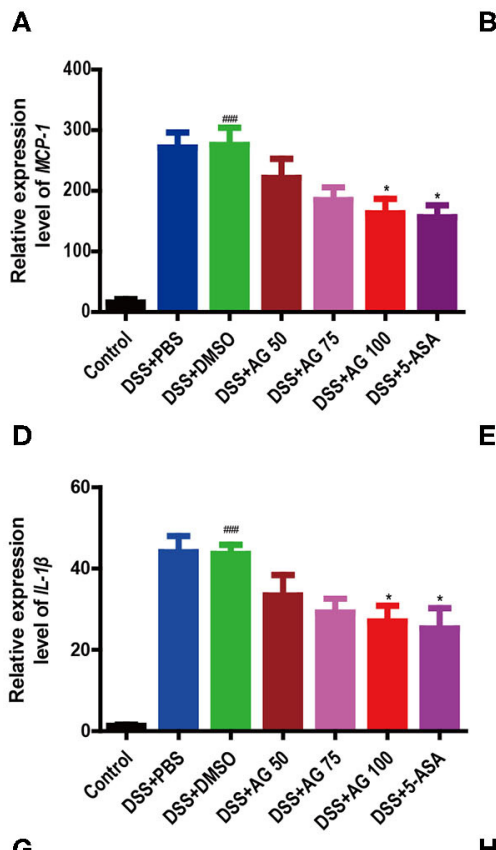

G

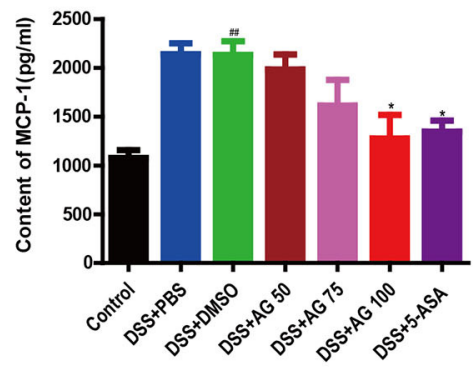

J

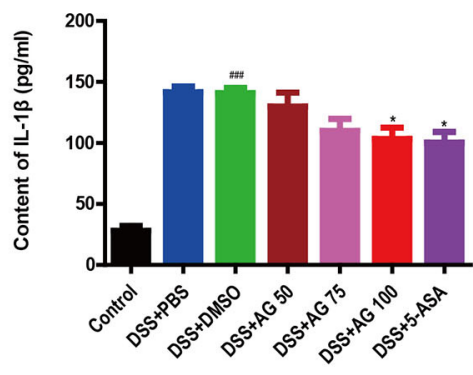

L

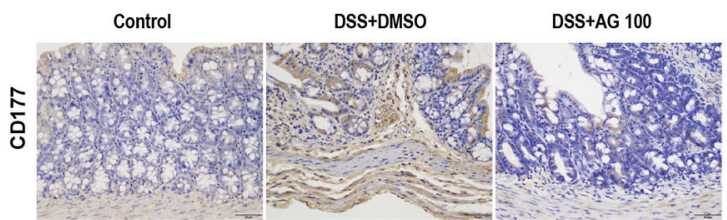

K
C
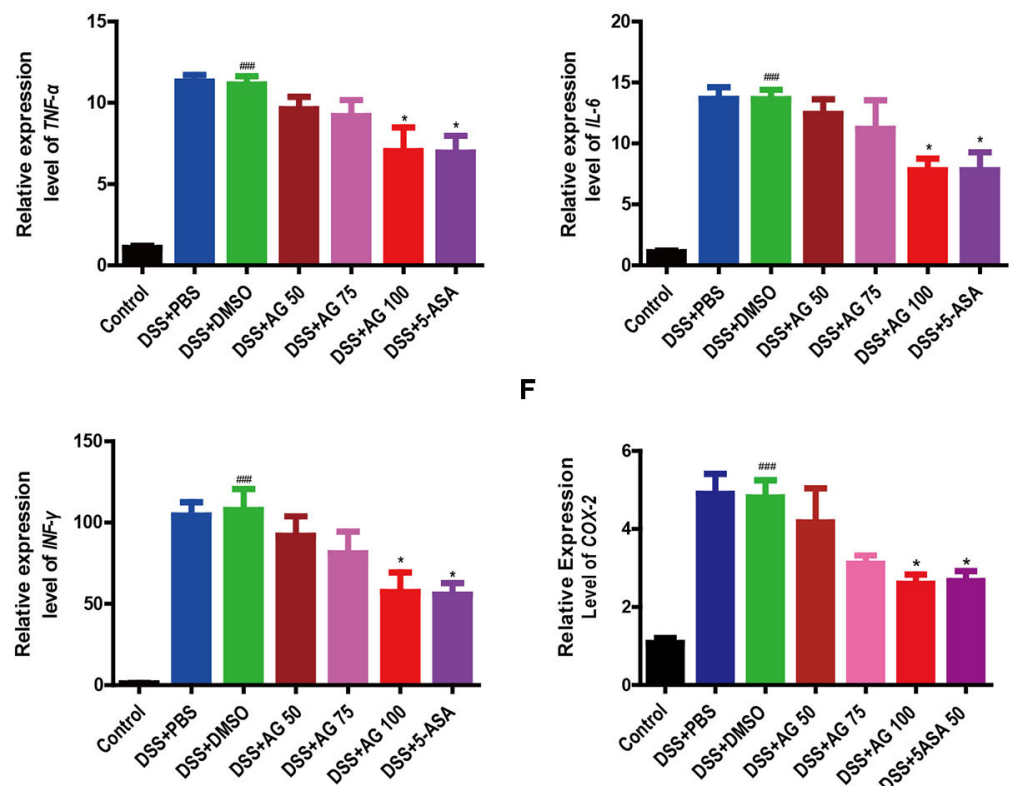

H
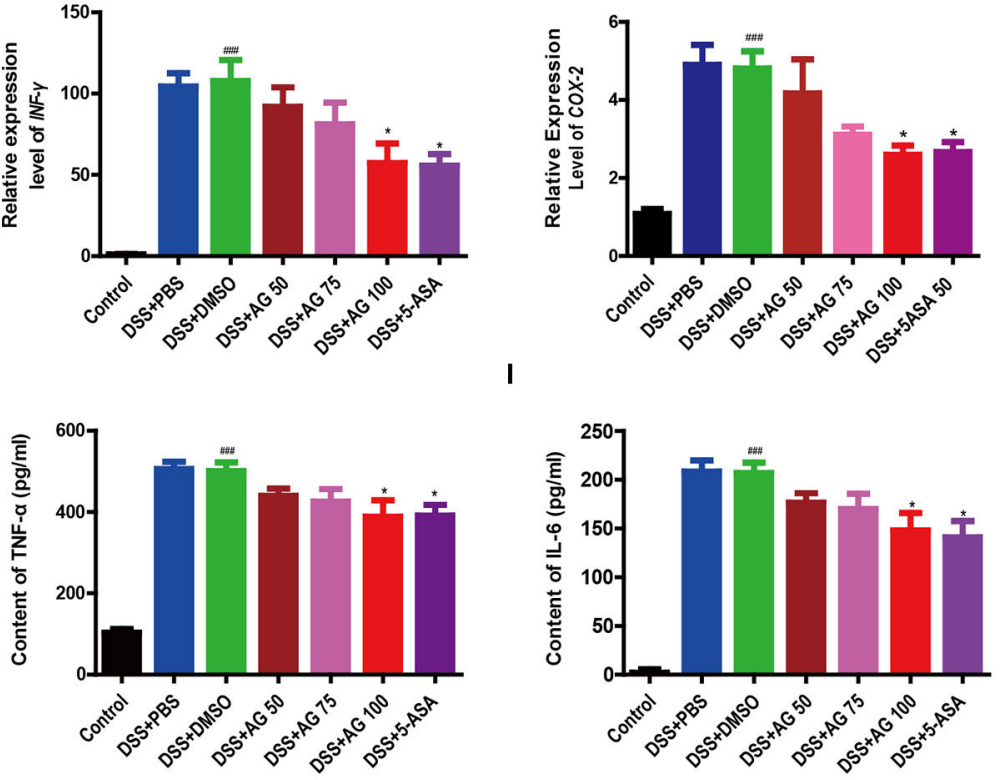

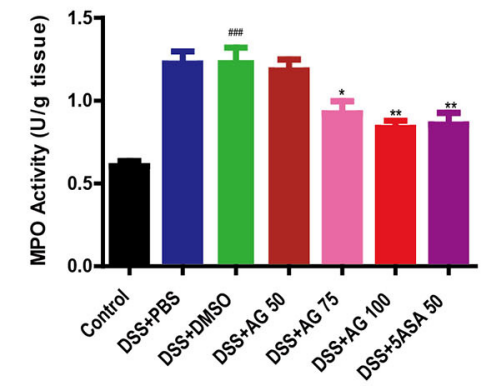

M

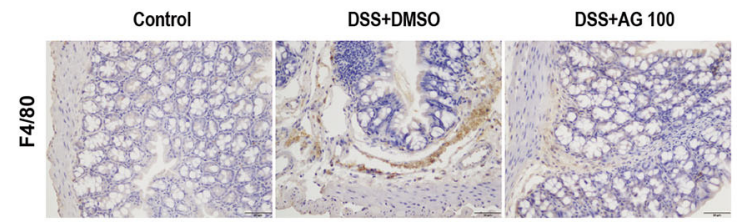

FIGURE 2 | Effects of AG on colon cytokines and the colonic infiltration of inflammatory cells in DSS-treated mice. Expression levels of (A) MCP-1, (B) TNF- $\alpha$, (C) $/ L-6$, (D) $/ L-1 \beta$, (E) IFN $\gamma$, and (F) COX-2 in the colon of acute colitis model mice determined by RT-qPCR. Colon tissue pro-inflammatory (G) MCP-1, (H) TNF- $\alpha$, (I) 
FIGURE 2 | IL-6, (J), and IL-1 $\beta$ levels. (K) MPO activity in the colon measured with an MPO assay kit. Data are expressed as the mean \pm SEM, $n=8$. Data were analyzed using one-way ANOVA (post hoc Tukey's multiple-comparison test) $\# \# P<0.01$ and $\# \# \# P<0.001$ compared with the control group. ${ }^{*} P<0.05$ and ${ }^{* *} P$ $<0.01$ compared with the DSS + DMSO group. Representative immunostaining images of colon sections stained for (L) CD177 and (M) F4/80. Formalin-fixed, paraffin-embedded $3 \mu \mathrm{m}$ cross-sections were stained with the appropriate primary antibody. Scale bar: $50 \mu \mathrm{m}$.

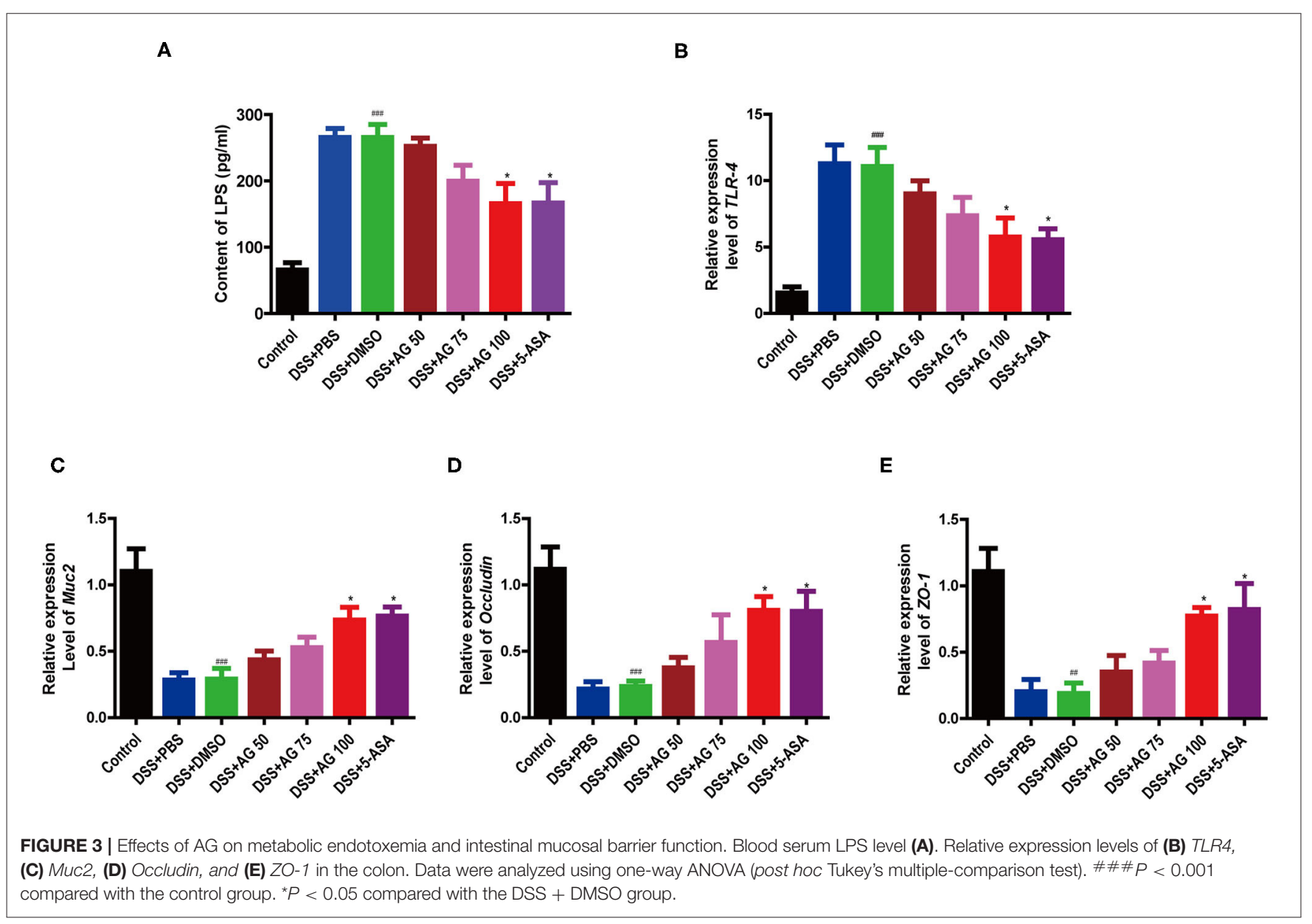

to the Lachnospiraceae family showed similar patterns as those in the Ruminococcaceae family (Figure S2H). Notably, the relative abundance of LPS-producing bacteria (Bacteroides and Escherichia-Shigella) was significantly increased in DSS-induced colitis mice, while AG inhibited this effect (Figures 5G,H).

Linear discriminant analysis effect size (LEfSe) analyses were used to explore which bacterium might be responsible for the impact on DSS-induced UC mice. The dominant microbiota at the family and genus levels for each group (Figure S2I and Figure 6A) were obtained. Then, a heatmap was constructed to display the 44 dominant microbiota in the 3 groups (Figures 6B-D). Specifically, 24 distinct families or genera were significantly reversed by AG treatment. Muribaculaceae, Ruminococcaceae, and Bacteroidaceae had the highest linear discriminant analysis (LDA) scores in the control group, the DSS+DMSO group, and the DSS+AG100 group, respectively, at the family level. Then, the correlations between Ruminococcaceae,
Escherichia-Shigella and LPS were analyzed by Pearson's correlation analysis (Figures 6E,F). Escherichia-Shigella and LPS showed a significant positive correlation (Pearson $r=0.7378, P<0.001)$. In contrast, Ruminococcaceae and LPS were negatively correlated (Pearson $r=-0.5233$, $P<0.05)$.

\section{DISCUSSION}

UC is well-known due to its high prevalence worldwide. In this study, a DSS-induced mouse model was used to simulate clinical UC. After a 7-day induction with 3\% DSS, the watery feces and decreasing body weights of the mice in the DSS + PBS and DSS + DMSO groups indicated successful model establishment. A series of studies have shown that flavonoids and their derivatives have good ameliorative effects on UC (18-20). We conducted the first study on the improvement of colitis by AG, a natural flavonoid 
A

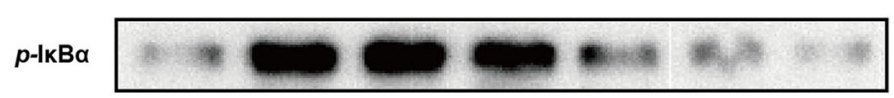

IKBa

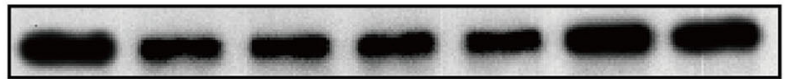

p-p65

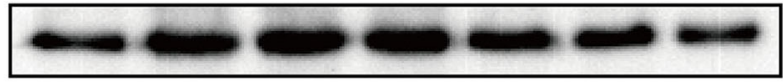

p65

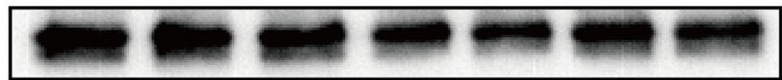

p-IKK $\alpha \beta$

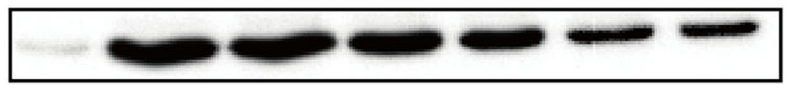

IKK $\alpha$

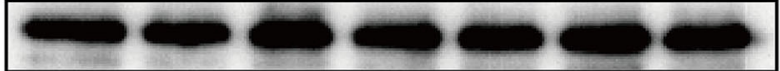

IKK $\beta$

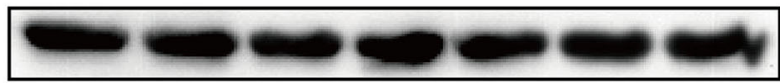

$\beta$-actin

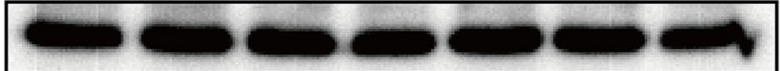

D

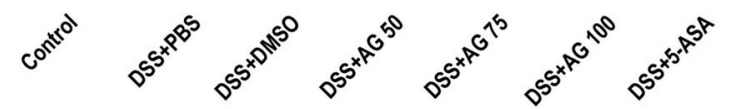

B

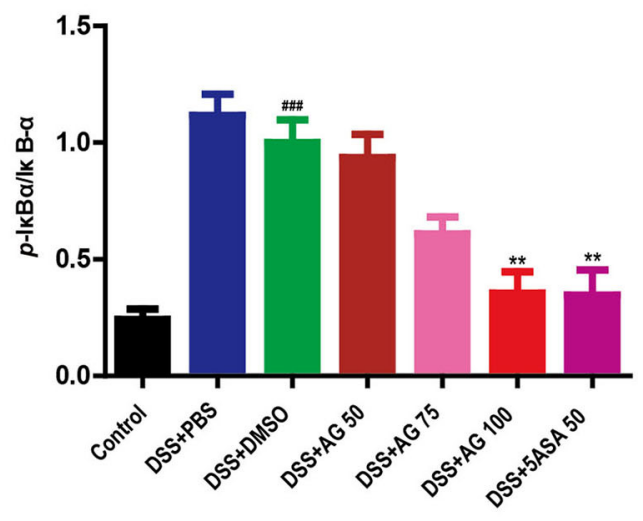

C

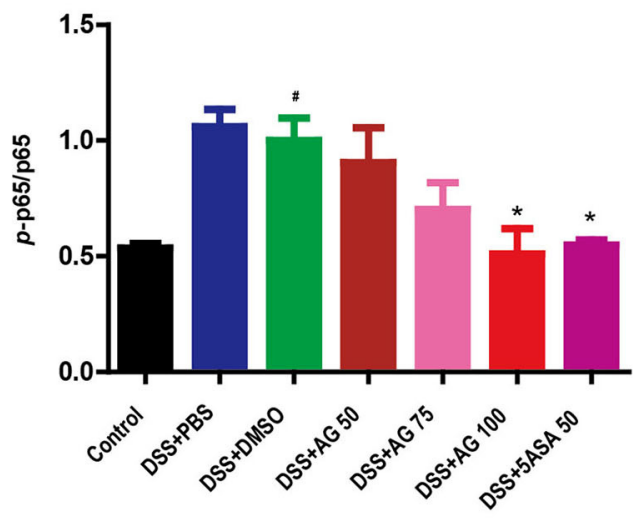

E

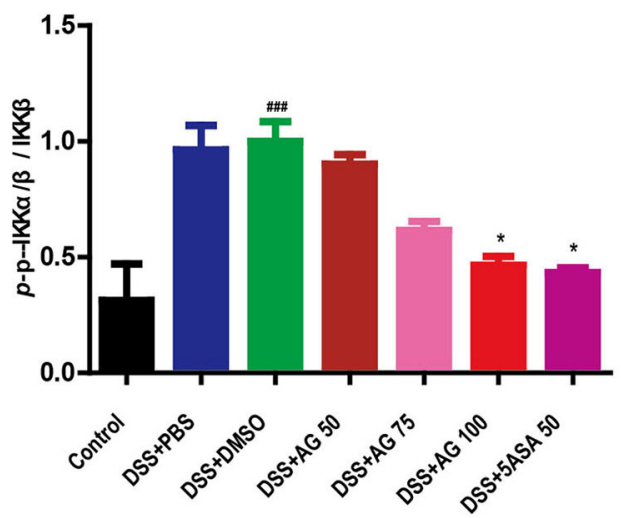

FIGURE 4 | AG suppressed the NF-kB signaling pathway. The phosphorylation of $1 \kappa B \alpha, p 65$, and IKK $\alpha / \beta$ was detected by Western blotting (A). (B-E) The relative density of each signaling band was calculated. $\beta$-actin was used as the protein loading control. Data were obtained from three independent experiments and are presented as the mean \pm SEM. Data were analyzed using one-way ANOVA (post hoc Tukey's multiple-comparison test). ${ }^{*} P<0.05$ and \#\#\# $P<0.001$ compared with the control group. ${ }^{*} P<0.05$ and ${ }^{* *} P<0.01$ compared with the DSS + DMSO group.

that is widely found in various medicinal and edible plants. Our results demonstrated that AG treatment could attenuate UC, as evidenced by the decreases in weight loss, colonic shortening,
DAI scores, and histological scores in the AG groups compared with the DSS group, and $100 \mathrm{mg} / \mathrm{kg} \cdot \mathrm{BW}$ AG had the best effect (Figure 1). 


\section{A}

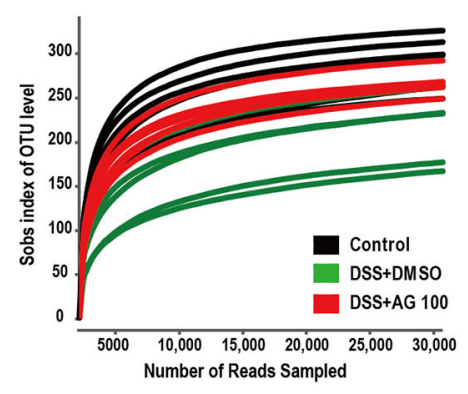

D

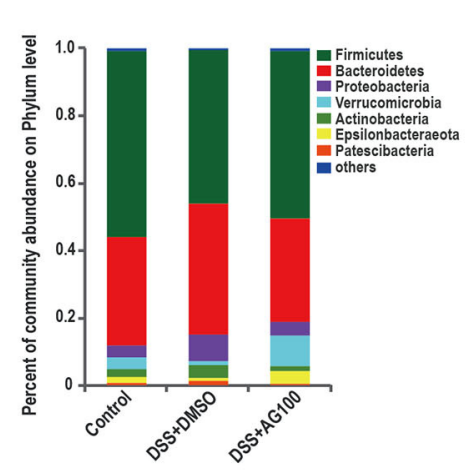

G
B

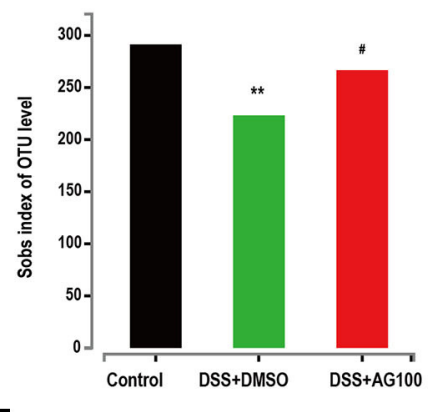

C

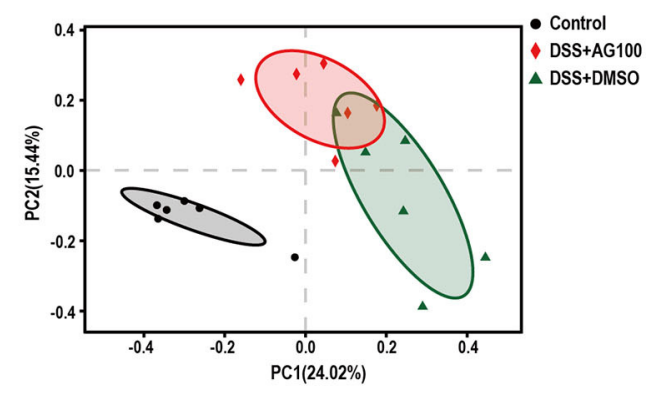

$\mathbf{F}$
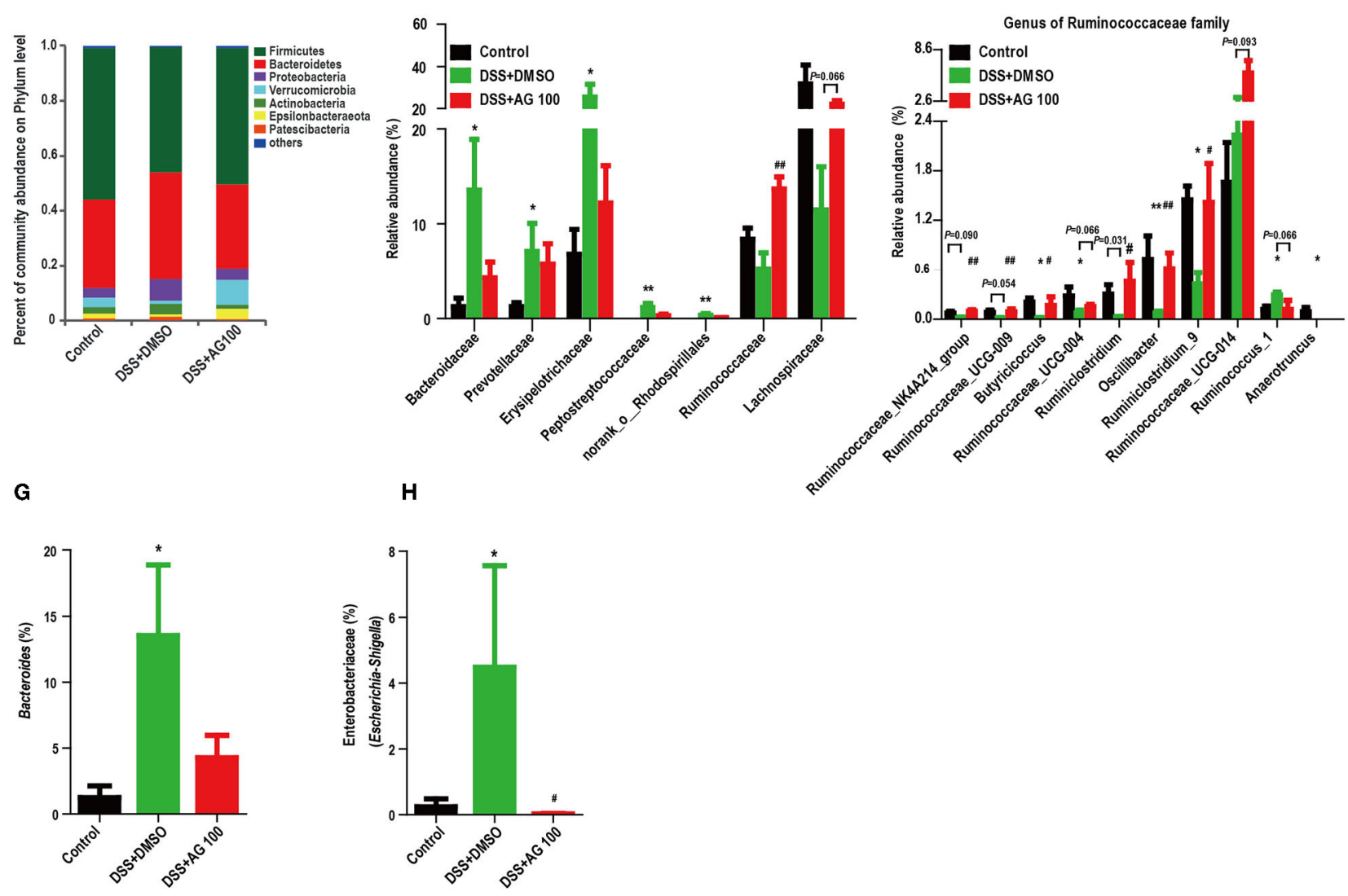

FIGURE 5 | AG altered the diversity and composition of the gut microbiota in colitis mice. (A) The rarefaction curve of the Sobs index of each sample plateau. (B) Alpha diversity estimated by the Sobs index. (C) PCoA plot based on the Bray-Curtis index. (D) Relative abundances of predominant gut microbiota at the phylum level. (E) Relative abundances of the gut microbial community at the family level. (F) Relative abundances of genera in the Ruminococcaceae family. (G) Relative abundances of Bacteroides species. (H) Relative abundances of Enterobacteriaceae (Escherichia-Shigella). * or \# indicates a significant difference between two groups using an unpaired two-tailed Student's $t$-test (alpha diversity) or the Wilcoxon rank-sum test (composition of the gut microbiota). ${ }^{*} P<0.05$ and ${ }^{\star \star} P<0.01$ compared with the control group. $\# P<0.05$ and $\# \# P<0.01$ compared with the DSS + DMSO group.

Reductions in inflammatory cytokine levels in the serum represent a logical goal for IBD therapy (21). The dramatically elevated mRNA expression levels of MCP-1, TNF- $\alpha$, IL6 , IL-1 $\beta$, and $I F N-\gamma$ in the colon are important features of the DSS colitis model $(21,22)$. In the present study, $100 \mathrm{mg} / \mathrm{kg} \cdot \mathrm{BW}$ AG significantly reduced the DSS-induced increases in the relative mRNA expression levels of $M C P$ 1 , TNF- $\alpha, I L-6, I L-1 \beta$, and IFN- $\gamma$ in the colon and the protein expression levels of pro-inflammatory factors in the colon, including MCP-1, TNF- $\alpha$, IL- 6 , and IL- $1 \beta$. The increased levels of pro-inflammatory cytokines in the colon of DSS-treated mice are usually caused by the infiltration of inflammatory cells. In the present study, increased colonic infiltration by macrophages and neutrophils was observed in DSS-induced colitis; AG treatment also improved this phenomenon (Figure 2). 
Lipopolysaccharide (LPS) is a cell-associated glycolipid that makes up the outer leaflet of the outer membrane of Gramnegative bacteria (23). LPS can promote the expression of proinflammatory cytokines and activate innate immune cells, such as macrophages, by stimulating signaling through $\operatorname{TLR} 4(24,25)$. Consistent with the effect of AG on the DSS-induced expression of pro-inflammatory cytokines in the colon, $100 \mathrm{mg} / \mathrm{kg} \cdot \mathrm{BW}$ AG significantly reduced the increased LPS content in the serum, $\mathrm{F} 4 / 80^{+}$macrophage infiltration and relative mRNA expression levels of TLR4 in colon tissues induced by DSS treatment in this study.

The intestinal epithelium, which is covered with a mucus gel layer, separates the luminal microbiota from systemic tissue (26). From the results for distal colonic sections, the mucus gel layer was completely damaged in the DSS group. Muc2 represents the main component of the colonic mucus. Deletion of Muc2 in mice eliminates mucus, causing bacterial colonization of the crypts and inflammation (26). DSS treatment significantly reduced Muc2 mRNA expression to a low level, while AG restored this expression to the level seen in the control group in the present study (Figure 3).

Maintaining the tightness and integrity of the intestinal barrier is also an important goal in the treatment of UC (21). The mRNA expression of intestinal tight junction-associated proteins ( $\mathrm{ZO}-1$ and Occludin) also suggested significant ameliorative effects for AG (Figure 3).

NF- $\kappa B$ is a key regulator of inflammation, innate immunity, and tissue integrity (27). The nuclear translocation of NF- $\mathrm{kB}$ is strongly activated in experimental colitis model animals and IBD patients (28). In the present study, the NF-kB signaling pathway was significantly activated in DSS-induced colitis mice.

Previous studies have shown that AG can inhibit the expression of lipopolysaccharide-induced inflammatory mediators in macrophages through the NF- $\mathrm{BB}$ pathway; AG can reduce mastitis (29), lung injury (30), uterine and epithelial inflammation (31), and colitis (32) in mice or rats by regulating the expression of NF- $\kappa \mathrm{B}$ signaling components. Consistent with previous studies, this study showed that AG suppressed the pro-inflammatory response by inhibiting the activation of the NF-kB pathway (Figure 4).

UC was previously thought to be an autoimmune disease but is now considered an infectious disease of the gut microbiota (33). In previous studies, DSS-treated mice were shown to exhibit dysbiosis of the gut microbiota, reflected as reductions in the numbers of symbionts and commensalists and/or an increase in the numbers of pathobionts. Reduced microbial diversity is a common phenomenon in UC (33). Herein, the microbiota community composition in the DSS-treated groups was significantly different from that in the control group. AG treatment improved the alpha diversity and changed the community composition.

At the phylum level, AG treatment reversed the DSSinduced changes in the relative abundances of Proteobacteria and Verrucomicrobia. Most Proteobacteria are thought to be harmful bacteria (33). Enterobacteriaceae (Escherichia-Shigella) is the typical family (genus) of Proteobacteria and includes LPSproducing, Gram-negative bacteria. Its relative abundance in the cecal content of DSS-induced colitis mice was increased more than 10 times than that in the cecal content of the mice in the control group; AG treatment significantly reduced its abundance to a level lower than that seen in the control group. It is worth noting that Escherichia-Shigella and LPS showed a significant positive correlation. This finding suggests that the ameliorative effect of AG on DSS-induced colitis is partly achieved by reducing the abundance of the classic pathogen genus Escherichia-Shigella. To date, Akkermansia is the only genus of bacteria belonging to Verrucomicrobia known to be in the gut. Emerging evidence has shown that reduced levels of Akkermansia are observed in IBD patients with inflammatory bowel diseases and metabolic disorders (34). In this study, AG reversed the reduction in Verrucomicrobia abundance in DSS-induced colitis mice.

At the family level, DSS treatment significantly increased the relative abundances of Bacteroidaceae, Prevotellaceae, Erysipelotrichaceae, Peptostreptococcaceae, and norank_o_Rhodospirillales and decreased the relative abundances of Ruminococcaceae and Lachnospiraceae, whereas AG treatment reversed these changes to varying degrees. In previous studies on UC, it was also shown that the abundances of colitogenic strains (Bacteroidaceae and Erysipelotrichaceae) are increased by induction with DSS $(33,35,36)$ and that their abundances are relatively high in patients with IBD $(37,38)$. Notably, most Bacteroidaceae and Erysipelotrichaceae bacteria have the ability to produce LPS. Ruminococcaceae and Lachnospiraceae have been recognized as probiotics that can produce SCFAs, such as $n$-butyric acid. DSS-induced UC animals $(39,40)$ and IBD patients $(41,42)$ always have lower abundances of Ruminococcaceae and Lachnospiraceae than healthy controls. Notably, Ruminococcaceae and LPS showed a significant negative correlation. Interestingly but puzzlingly, DSS increased the abundances of Prevotellaceae and Peptostreptococcaceae, which are thought to be potentially beneficial families.

At the genus level (Figure 6), Family_XIII_UCG-001, Unclassified $f$ _ Lachnospiraceae, Ruminiclostridium_9, Oscillibacter, Butyricicoccus, Ruminiclostridium, Ruminococcaceae_NK4A214_group, Ruminococcaceae_ UCG-009, and Peptococcus showed low relative abundances in the DSS + DMSO group, patterns that were significantly reversed by AG $(P<0.05)$. Among these genera, Ruminiclostridium_9, Oscillibacter, Butyricicoccus, Ruminiclostridium, Ruminococcaceae_NK4A214_group, and Ruminococcaceae_UCG-009 all belong to the Ruminococcaceae family. These reductions are characteristics of the gut microbiota in DSS-treated mice that were observed in previous studies (43-45).

Notably, Oscillibacter is associated with severe DSS-induced colitis $(21,46)$. B. pullicaecorum (a species of Butyricicoccus) could attenuate TNBS-induced colitis in rats, and a supernatant derived from a B. pullicaecorum culture could strengthen epithelial barrier function. Our results demonstrated that the ameliorative effects of AG on DSS-induced UC symptoms were achieved by changing the gut microbiota, especially certain specific microbiomes, and we concluded that Ruminococcaceae and Enterobacteriaceae (Escherichia-Shigella) might be the key groups affected by AG to improve UC.

In conclusion, AG exerted protective effects against UC through several targets, including metabolic endotoxemia (serum 

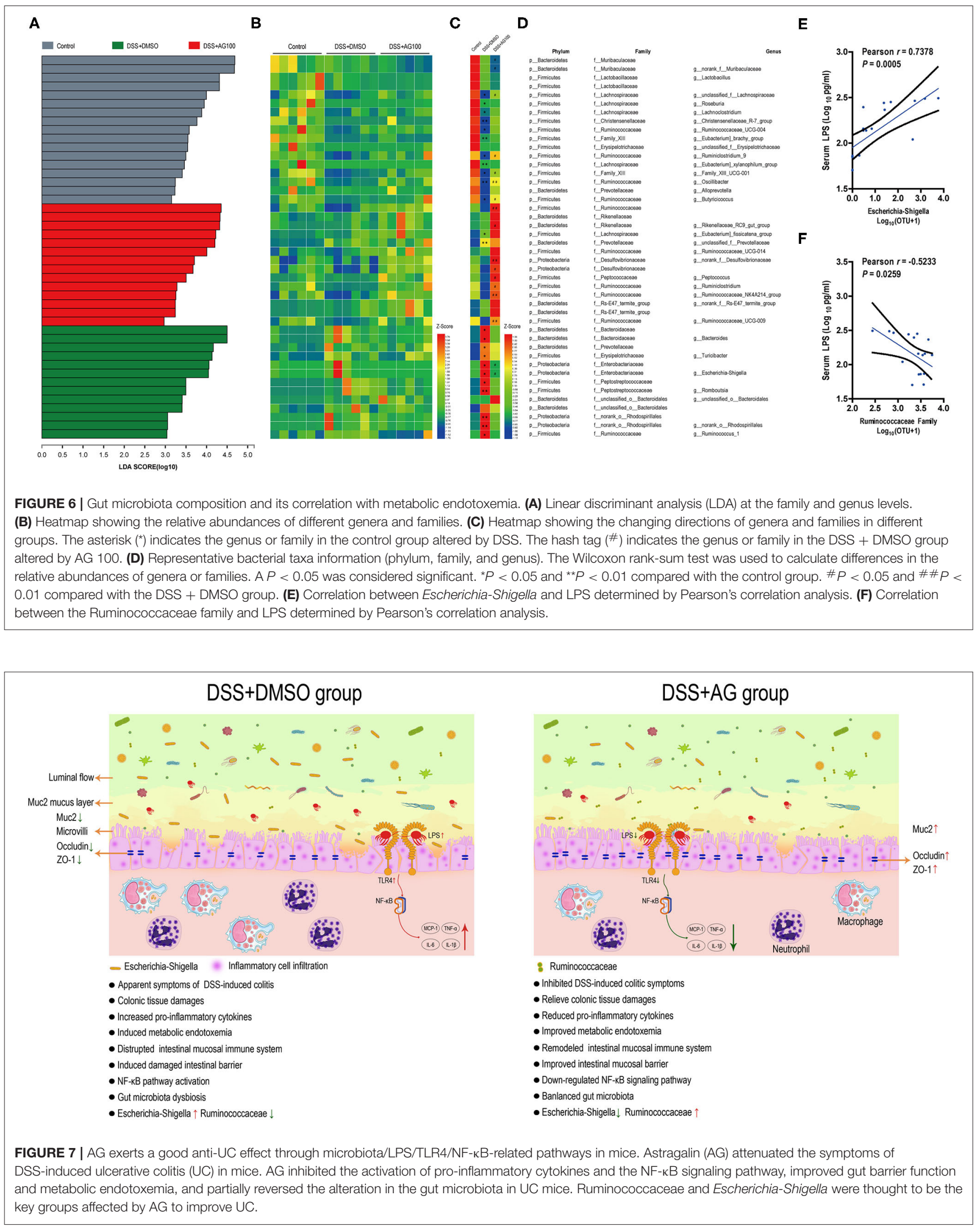
LPS), pro-inflammatory cytokines (COX-2, MCP-1, TNF- $\alpha$, IL-6, IL- $1 \beta$, and IFN- $\gamma$ ), enhancement of the mRNA expression levels of mucin (Muc2) and tight junction proteins ( $\mathrm{ZO}-1$ and occludin) in the colon, inhibition of the relative mRNA expression levels of TLR4, inhibition of NF- $\mathrm{B}$ pathway activation and modulation of the gut microbiota. Through systematic data analysis and literature review, key bacteria related to UC were identified (Ruminococcaceae and Escherichia-Shigella). In summary, AG might exert good anti-inflammatory effects by affecting the microbiota/LPS/TLR4/NF-кB-related pathway (Figure 7).

\section{DATA AVAILABILITY STATEMENT}

The datasets generated for this study can be found in online repositories. The names of the repository/repositories and accession number(s) can be found at: https://www.ncbi.nlm.nih. gov/genbank/, PRJNA578013.

\section{ETHICS STATEMENT}

The animal study was reviewed and approved by Yunnan Agricultural University Animal Ethics Committee with respect to ethical issues and scientific care (YNAU-2018-031).

\section{REFERENCES}

1. Torres J, Mehandru S, Colombel JF, Peyrin-Biroulet L. Crohn's disease. Lancet. (2016) 389:822-4. doi: 10.1016/S0140-6736(16)31711-1

2. Ungaro R, Mehandru S, Allen PB, Peyrinbiroulet L, Colombel JF. Ulcerative colitis. Lancet. (2016) 2:340-1. doi: 10.1016/S0140-6736(16)32126-2

3. Ananthakrishnan AN, Bernstein CN, Iliopoulos D, Macpherson A, Neurath MF, Rar A, et al. Environmental triggers in IBD: a review of progress and evidence. Nat Rev Gastroenterol Hepatol. (2018) 15:39-49. doi: 10.1038/nrgastro.2017.136

4. Zhang Z, Wu X, Cao S, Wang L, Wang D, Yang H, et al. Caffeic acid ameliorates colitis in association with increased Akkermansia population in the gut microbiota of mice. Oncotarget. (2016) 7:31790-9. doi: 10.18632/oncotarget.9306

5. Riaz A, Rasul A, Hussain G, Zahoor MK, Jabeen F, Subhani Z, et al. Astragalin: a bioactive phytochemical with potential therapeutic activities. Adv Pharmacol Sci. (2018) 2018:1-15. doi: 10.1155/2018/9794625

6. Wang Z, Li Q, Xiang M, Zhang F, Wei D, Wen Z, et al. Astragaloside alleviates hepatic fibrosis function via PAR2 signaling pathway in diabetic rats. Cell Biochem Int J Exp Cell Physiol Biochem Pharmacol. (2017) 41:1156-66. doi: 10.1159/000464122

7. Usune E, Alfredo FQ, Milagro FI, Leixuri AJ, Alfredo M, et al. Impact of polyphenols and polyphenol-rich dietary sources on gut microbiota composition. J Agric Food Chem. (2013) 61:9517-33. doi: 10.1021/jf402506c

8. Taylor RA, Leonard MC. Curcumin for inflammatory bowel disease: a review of human studies. Altern Med Rev. (2011) 16:152-6.

9. Dodda D, Chhajed R, Mishra J. Protective effect of quercetin against acetic acid induced inflammatory bowel disease (IBD) like symptoms in rats: possible morphological and biochemical alterations. Pharmacol Rep. (2014) 66:169-73. doi: 10.1016/j.pharep.2013.08.013

10. Yao J, Wei C, Wang JY, Zhang R, Li YX, Wang LS. Effect of resveratrol on Treg/Th17 signaling and ulcerative colitis treatment in mice. World $J$ Gastroenterol. (2015) 21:6572-81. doi: 10.3748/wjg.v21.i21.6572

11. Bitzer ZT, Elias RJ, Vijay-Kumar M, Lambert JD. (-)-Epigallocatechin-3gallate decreases colonic inflammation and permeability in a mouse model of colitis, but reduces macronutrient digestion and exacerbates weight loss. Mol Nutr Food Res. (2016) 60:2267-74. doi: 10.1002/mnfr.201501042

\section{AUTHOR CONTRIBUTIONS}

YT and JS designed the experiments. LP, XG, and LN performed the animal studies. LP, XG, LN, YW, and JX performed the molecular biology experiments. XG, LP, CS, and LT analyzed the 16S rRNA gene sequencing data. LP, XG, YT, and JS prepared the manuscript and had primary responsibility for the final content. All authors read and approved the final manuscript.

\section{FUNDING}

This research was funded by the Major Project of the Science and Technology Department of Yunnan Province (2018ZI001 and 2019ZG00905) and the Personnel Training Project on Academic and Technical Leaders of Yunnan Province (2018HB040).

\section{SUPPLEMENTARY MATERIAL}

The Supplementary Material for this article can be found online at: https://www.frontiersin.org/articles/10.3389/fimmu. 2020.02058/full\#supplementary-material
12. Chen T, Hu S, Zhang H, Guan Q, Yang Y, Wang X. Anti-inflammatory effects of Dioscorea alata L. anthocyanins in a TNBS-induced colitis model. Food Funct. (2017) 8:659-69. doi: 10.1039/C6FO01273F

13. Tremaroli V, Bäckhed F. Functional interactions between the gut microbiota and host metabolism. Nature. (2012) 489:242-9. doi: 10.1038/nature11552

14. Stefan W, Clemens N, Benno W, Neurath MF. Chemically induced mouse models of intestinal inflammation. Nat Protoc. (2007) 2:541-6. doi: $10.1038 /$ nprot.2007.41

15. Cooper H. Clinicopathologic study of dextran sulfate sodium experimental murine colitis. Lab Investig. (1993) 69:238-49.

16. Stillie R, Stadnyk AW. Role of TNF receptors, TNFR1 and TNFR2, in dextran sodium sulfate-induced colitis. Inflamm Bowel Dis. (2009) 15:151525. doi: 10.1002/ibd.20951

17. Huang $Y$, Zhu Q, Yang $X, X u ~ H$, Sun B, Wang X, et al. Wound healing can be improved by (-)-epigallocatechin gallate through targeting Notch in streptozotocin-induced diabetic mice. FASEB J. (2018) 33:953-64. doi: 10.1096/fj.201800337R

18. Tao F, Qian C, Guo W, Luo Q, Xu Q, Sun Y. Inhibition of Th1/Th17 responses via suppression of STAT1 and STAT3 activation contributes to the amelioration of murine experimental colitis by a natural flavonoid glucoside icariin. Biochem Pharmacol. (2013) 85:798-807. doi: 10.1016/j.bcp.2012.12.002

19. Sahu BD, Kumar JM, Sistla R. Fisetin, a dietary flavonoid, ameliorates experimental colitis in mice: relevance of NF-кB signaling. J Nutr Biochem. (2015) 28:171-82. doi: 10.1016/j.jnutbio.2015.10.004

20. Zhao Y, Guo Q, Zhu Q, Tan R, Bai D, Bu X, et al. Flavonoid VI-16 protects against DSS-induced colitis by inhibiting Txnip-dependent NLRP3 inflammasome activation in macrophages via reducing oxidative stress. Mucosal Immunol. (2019) 12:1150-63. doi: 10.1038/s41385-019-0177-x

21. Peng Y, Yan Y, Wan P, Chen D, Ding Y, Ran L, et al. Gut microbiota modulation and anti-inflammatory properties of anthocyanins from the fruits of Lycium ruthenicum Murray in dextran sodium sulfate-induced colitis in mice. Free Radic Biol Med. (2019) 136:96-108. doi: 10.1016/j.freeradbiomed.2019.04.005

22. Shin HS, Satsu H, Bae M, Zhao Z, Ogiwara H, Totsuka M, et al. Antiinflammatory effect of chlorogenic acid on the IL- 8 production in Caco- 2 cells and the dextran sulphate sodium-induced colitis symptoms in C57BL/6 mice. Food Chem. (2015) 168:167-75. doi: 10.1016/j.foodchem.2014.06.100 
23. Jacobson AN, Choudhury BP, Fischbach MA. The biosynthesis of lipooligosaccharide from Bacteroides thetaiotaomicron. MBio. (2018) 9:e02289-17. doi: 10.1128/mBio.02289-17

24. Lorenz E, Patel DD, Hartung T, Schwartz DA. Toll-Like Receptor 4 (TLR4)deficient murine macrophage cell line as an in vitro assay system to show TLR4-independent signaling of Bacteroides fragilis lipopolysaccharide. Infect Immun. (2002) 70:4892-6. doi: 10.1128/IAI.70.9.4892-4896.2002

25. Mancuso G, Midiri A, Biondo C, Beninati C, Gambuzza M, Macri D, et al. Bacteroides fragilis-derived lipopolysaccharide produces cell activation and lethal toxicity via toll-like receptor 4. Infect Immun. (2005) 73:5620-7. doi: 10.1128/IAI.73.9.5620-5627.2005

26. Birchenough GMH, Nystrom EEL, Johansson MEV, Hansson GC. A sentinel goblet cell guards the colonic crypt by triggering Nlrp6-dependent Muc2 secretion. Science. (2016) 352:1535-42. doi: 10.1126/science.aaf7419

27. Banoth B, Chatterjee B, Vijayaragavan B, Prasad M, Roy P, Basak S. Stimulus-selective crosstalk via the NF- $\kappa \mathrm{B}$ signaling system reinforces innate immune response to alleviate gut infection. ELife. (2015) 4:e05648. doi: 10.7554/eLife.05648.029

28. Atreya I, Atreya R, Neurath MF. NF-kappaB in inflammatory bowel disease. $J$ Intern Med. (2008) 263:591. doi: 10.1111/j.1365-2796.2008.01953.x

29. Li F, Liang D, Yang Z, Wang T, Wang W, Song X, et al. Astragalin suppresses inflammatory responses via down-regulation of NF- $\mathrm{B}$ signaling pathway in lipopolysaccharide-induced mastitis in a murine model. Int Immunopharmacol. (2013) 17:478-82. doi: 10.1016/j.intimp.2013.07.010

30. Soromou LW, Chen N, Jiang L, Huo M, Wei M, Chu X, et al. Astragalin attenuates lipopolysaccharide-induced inflammatory responses by downregulating NF- $\mathrm{B}$ signaling pathway. Biochem Biophys Res Commun. (2012) 419:256-61. doi: 10.1016/j.bbrc.2012.02.005

31. Zhang W, Lu X, Wang W, Ding Z, Fu Y, Zhou X, et al. Inhibitory effects of emodin, thymol, and astragalin on leptospira interrogans-induced inflammatory response in the uterine and endometrium epithelial cells of mice. Inflammation. (2017) 40:666-75. doi: 10.1007/s10753-017-0513-9

32. Han YM, Koh J, Kim JH, Lee J, Im JP, Kim JS. Astragalin inhibits nuclear factor- $\mathrm{\kappa} \mathrm{B}$ signaling in human colonic epithelial cells and attenuates experimental colitis in mice. Gut Liver. (2020). doi: 10.5009/gnl19268. [Epub ahead of print]

33. Ohkusa $\mathrm{T}$, Koido S. Intestinal microbiota and ulcerative colitis. I Infect Chemother. (2015) 21:761-8. doi: 10.1016/j.jiac.2015.07.010

34. Derrien M, Belzer C, de Vos WM. Akkermansia muciniphila and its role in regulating host functions. Microb Pathog. (2017) 106:171-81. doi: 10.1016/j.micpath.2016.02.005

35. Chen L, Wilson JE, Koenigsknecht MJ, Chou W, Montgomery SA, Truax AD, et al. Corrigendum: NLRP12 attenuates colon inflammation by maintaining colonic microbial diversity and promoting protective commensal bacterial growth. Nat Immunol. (2017) 18:1270. doi: 10.1038/ni1117-1270b

36. Toshifumi O, Eri M, Shunichi A, Yasuhiro D, Junji Y, Jun K, et al. Metaanalysis of fecal microbiota and metabolites in experimental colitic mice during the inflammatory and healing phases. Nutrients. (2017) 9:1329. doi: 10.3390/nu9121329
37. Frank DN, Amand ALS, Feldman RA, Boedeker EC, Pace NR. Molecularphylogenetic characterization of microbial community imbalances in human inflammatory bowel diseases. Proc Natl Acad Sci USA. (2007) 104:13780-5. doi: $10.1073 /$ pnas.0706625104

38. Walker AW, Sanderson JD, Churcher C, Parkes GC, Hudspith BN, Rayment $\mathrm{N}$, et al. High-throughput clone library analysis of the mucosa-associated microbiota reveals dysbiosis and differences between inflamed and noninflamed regions of the intestine in inflammatory bowel disease. BMC Microbiol. (2011) 11:7. doi: 10.1186/1471-2180-11-7

39. Kim H, Banerjee N, Sirven MA, Minamoto Y, Markel ME, Suchodolski JS, et al. Pomegranate polyphenolics reduce inflammation and ulceration in intestinal colitis-involvement of the miR-145/p70S6K1/HIF $1 \alpha$ axis in vivo and in vitro. J Nutr Biochem. (2017) 43:107-15. doi: 10.1016/j.jnutbio.2017.02.005

40. Pei L, Ke Y, Zhao H, Wang L, Jia C, Liu W, et al. Role of colonic microbiota in the pathogenesis of ulcerative colitis. BMC Gastroenterol. (2019) 19:10-1. doi: 10.1186/s12876-019-0930-3

41. Tyler AD, Kirsch R, Milgrom R, Stempak JM, Kabakchiev B, Silverberg MS. Microbiome heterogeneity characterizing intestinal tissue and inflammatory bowel disease phenotype. Inflamm Bowel Dis. (2016) 22:807-16. doi: 10.1097/MIB.0000000000000674

42. Yilmaz B, Juillerat P, Øyås O, Ramon C, Bravo FD. Franc Y, et al. Publisher Correction: Microbial network disturbances in relapsing refractory Crohn's disease. Nat Med. (2019) 25:701. doi: 10.1038/s41591-0190411-9

43. Quraishi MN, Sergeant M, Kay G, Iqbal T, Chan J, Constantinidou C, et al. The gut-adherent microbiota of PSC-IBD is distinct to that of IBD. Gut. (2017) 66:381-6. doi: 10.1136/gutjnl-2016-311915

44. Liu G, Yan W, Ding S, Jiang H, Ma Y, Wang H, et al. Effects of IRW and IQW on oxidative stress and gut microbiota in dextran sodium sulfateinduced colitis. Cell Physiol Biochem. (2018) 51:441-51. doi: 10.1159/0004 95240

45. Wang C, Li W, Wang H, Ma Y, Zhao X, Yang H, et al. VSL\#3 can prevent ulcerative colitis-associated carcinogenesis in mice. World J Gastroenterol. (2018) 24:4254-62. doi: 10.3748/wjg.v24.i37.4254

46. Li M, Wu Y, Hu Y, Zhao L, Zhang C. Initial gut microbiota structure affects sensitivity to DSS-induced colitis in a mouse model. Sci China Life Sci. (2018) 61:762-9. doi: 10.1007/s11427-017-9097-0

Conflict of Interest: The authors declare that the research was conducted in the absence of any commercial or financial relationships that could be construed as a potential conflict of interest.

Copyright (c) 2020 Peng, Gao, Nie, Xie, Dai, Shi, Tao, Wang, Tian and Sheng. This is an open-access article distributed under the terms of the Creative Commons Attribution License (CC BY). The use, distribution or reproduction in other forums is permitted, provided the original author(s) and the copyright owner(s) are credited and that the original publication in this journal is cited, in accordance with accepted academic practice. No use, distribution or reproduction is permitted which does not comply with these terms. 